\title{
Kinematical Brownian motion of the harmonic oscillator in non-commutative space
}

\author{
Martin Tchoffo ${ }^{1}$, Jules Casimir Ngana Kuetche ${ }^{1,2}$, Georges Collince Fouokeng ${ }^{1}$, \\ Ngwa Engelbert Afuoti ${ }^{1,3}$, Lukong Cornelius Fai ${ }^{1}$ \\ ${ }^{1}$ Mesoscopic and Multilayer Structures Laboratory, Department of Physics, University of Dschang, Cameroon \\ ${ }^{2}$ Department of Physics, University of Buea, Buea, Cameroon \\ ${ }^{3}$ Department of Thermal Engineering and Energetic, Douala University Institute of Technology, Douala, Cameroon
}

Email address:

mtchoffo2000@yahoo.fr (M. Tchoffo)

\section{To cite this article:}

Martin Tchoffo, Jules Casimir Ngana Kuetche, Georges Collince Fouokeng, Ngwa Engelbert Afuoti, Lukong Cornelius Fai. Kinematical Brownian Motion of the Harmonic Oscillator in Non-Commutative Space. American Journal of Modern Physics.

Vol. 3, No. 3, 2014, pp. 138-142. doi: 10.11648/j.ajmp.20140303.14

\begin{abstract}
In this work the Jacobi's second equality in the form of stochastic equation and the Wiener path integral approach are used to evaluate the probability density of harmonic oscillator in non-commutative space. Using the factorization theorem and the Mastubara formalism, the thermodynamic parameters are determined. The structure of Fokker-Planck equation remained the same even in a commutative and non-commutative space. Moreover, the noncommutative parameter is depicted for increasing value of the entropy.
\end{abstract}

Keywords: Brownian Motion, Stochastic Equation, Wiener Process, Fokker-Planck Equation, Non-Commutative Space

\section{Introduction}

Recently, the effect of random forces on the motion of a harmonic oscillator has been studied with much interest. Thus, considerable attention has been made in this field to understand the process of Euclidian quantum mechanics in the theory of dynamic Brownian motion [1,2].This is explained by the close relationship between the Brownian motion and quantum mechanics [3-6].

When solving the problem of ultraviolet divergences, Heisenberg and Schrödinger conjectured that space-time coordinates may be mutually non-commutative[7].This was the starting point of several researches on the development and the applications of non-commutative space in string theory, cosmology, and more recently quantum hall effect, spin-orbit interaction and in quantum dots[7-10]. The noncommutative analogue of the random variable, random walk, Gaussian, Levis, Ito, and Markovian processes seem to be the most investigated class of stochastic process in physics when studying probability theory and has been of great concern over the last twenty years [10-15].

In the present work, with the help of the Jacobi's second equality in the form of stochastic equation in a noncommutative space, some thermodynamic parameters of a harmonic oscillator are determined using the theorem of factorization and Mastubara formalism. The organization of the work is as follows: section II presents the kinematical Brownian motion and its link with the Euclidean quantum mechanics. In Section 3, in the light of the factorization theorem and the Mastubara formalism, the entropy and the specific heat capacity are determined. We then close the work with the conclusion.

\section{Kinematical Brownian Motion}

Considering the following Jacobi's second equality in the form of stochastic equation [1],

$$
\dot{x}_{\tau}+\frac{1}{m} \frac{\partial S_{c l}\left(x_{\tau}, \tau ; x, t\right)}{\partial x_{\tau}}=\dot{\varphi}(\tau)
$$

The corresponding Fokker-Planck equation associated to the stochastic equation (1) is: 


$$
\hbar \frac{\partial W}{\partial \tau}-\frac{\hbar}{m} \frac{\partial}{\partial x_{\tau}}\left(\frac{\partial S_{c l}\left(x_{\tau}, \tau ; x, t\right)}{\partial x_{\tau}} W\right)=\frac{\hbar^{2}}{2 m} \frac{\partial^{2} W}{\partial x_{\tau}^{2}}
$$

Using the factorization theorem, the solution of equation (2) yields:

$$
W\left(x_{0}, 0 ; x_{\tau}, \tau ; x, t\right)=\frac{\exp \left[-\frac{1}{\hbar} S_{c l}\left(x_{\tau}, \tau ; x, t\right)\right]}{\exp \left[-\frac{1}{\hbar} S_{c l}\left(x_{0}, 0 ; x, t\right)\right]} Z\left(x_{0}, 0 ; x_{\tau}, \tau\right)
$$

where

$$
Z\left(x_{0}, 0 ; x_{\tau}, \tau\right)=\int_{C} \exp \left[-\int_{0}^{\tau}\left(V(x(s), s)-\frac{1}{2 m} \frac{\partial^{2} S_{c l}(x(s), s ; x, t)}{\partial x_{s}^{2}}\right) d s\right] d_{w} x_{s}
$$

is the Kac's formula with

$$
x(0)=x_{0}, x(t)=x, x(\tau)=x_{\tau}
$$

From Eq. (3), the solution of the Bloch equation is derived as:

$$
\frac{\hbar \partial \exp \left[-\frac{1}{\hbar} S_{c l}\left(x_{\tau}, \tau ; x, t\right)\right]}{\partial \tau}=\hat{\mathrm{H}} \exp \left[-\frac{1}{\hbar} S_{c l}\left(x_{\tau}, \tau ; x, t\right)\right]
$$

Which demonstrate that the Brownian motion is related to Euclidean quantum mechanics [1].

$$
\left\{\begin{array}{l}
\dot{x}_{1 \tau}+\frac{w}{\sqrt{k} \sin (w \sqrt{k} T)}\left[x_{1 \tau} \cos (w \sqrt{k} T)-x_{1}^{\prime \prime} \cos (w \sqrt{k-1} T)+x_{2}^{\prime \prime} \sin (w \sqrt{k-1} T)\right]=\dot{\varphi}(\tau) \\
\dot{x}_{2 \tau}+\frac{w}{\sqrt{k} \sin (w \sqrt{k} T)}\left[x_{2 \tau} \cos (w \sqrt{k} T)-x_{2}^{\prime \prime} \cos (w \sqrt{k-1} T)+x_{1}^{\prime \prime} \sin (w \sqrt{k-1} T)\right]=\dot{\varphi}(\tau)
\end{array}\right.
$$

where $x_{1}^{\prime}=x_{1 \tau} ; x_{2}^{\prime}=x_{2 \tau}$

Using the canonical transformation, the solution of Fokker-Planck equation associated to (7) is:

$$
W_{\theta}^{H}\left(x_{0}, 0 ; x_{\tau}, \tau ; x, t\right)=\left[\frac{(2 \pi \hbar \sin (w \sqrt{k} \tau))^{2 k} \sin (w \sqrt{k}(t-\tau))}{(m w)^{2 k} \sin (w \sqrt{k} t)}\right]^{-\frac{1}{2 k}} \exp \left\{\frac{1}{2} \eta\left[\begin{array}{l}
\xi_{1}^{2}\left(x_{10}, 0 ; x_{1 \tau}, \tau ; x_{1}^{\prime \prime}, t\right) \\
\xi_{2}^{2}\left(x_{20}, 0 ; x_{2 \tau} ; \tau, x_{2}^{\prime \prime}, t\right) \\
\chi\left(x_{0}, 0 ; x_{\tau}, \tau ; x^{\prime \prime}, t\right)
\end{array}\right]\right\}
$$

where:

$$
\begin{array}{r}
\xi_{1}\left(x_{10}, 0 ; x_{1 \tau}, \tau ; x_{1}^{\prime \prime}, t\right)=x_{1 \tau}-\frac{x_{1}^{\prime \prime}-x_{10} \cos (w \sqrt{k} \tau)}{\sin (w \sqrt{k} t)} \sin (w \sqrt{k} \tau)-x_{10} \cos (w \sqrt{k} t) \\
\xi_{2}\left(x_{20}, 0 ; x_{2 \tau}, \tau ; x_{2}^{\prime \prime}, t\right)=x_{2 \tau}-\frac{x_{2}^{\prime \prime}-x_{20} \cos (w \sqrt{k} \tau)}{\sin (w \sqrt{k} t)} \sin (w \sqrt{k} \tau)-x_{20} \cos (w \sqrt{k} t)
\end{array}
$$




$$
\begin{aligned}
\chi\left(x_{0}, 0 ; x_{\tau}, \tau ; x, t\right)= & {\left[\begin{array}{l}
\left.\left(x_{10} x_{1 \tau}+x_{20} x_{2 \tau}\right)(\cos (w \sqrt{k-1} \tau)-1)+\left(x_{1 \tau} x_{1}^{\prime \prime}+x_{2 \tau} x_{2}^{\prime \prime}\right) \cdot\right] \\
(1-\cos w \sqrt{k-1}(t-\tau))+ \\
\cos w \sqrt{k} \tau(\cos w \sqrt{k-1} t-1)\left(x_{10} x_{1}^{\prime \prime}+x_{20} x_{2}^{\prime \prime}\right)
\end{array}\right] \frac{\sin (w \sqrt{k-1} \tau)}{\sin (w \sqrt{k} t)}+} \\
& (1-\cos w \sqrt{k-1} t)\left(x_{10} x_{1}^{\prime \prime}+x_{20} x_{2}^{\prime \prime}\right)\left(\frac{\sin w \sqrt{k-1} \tau}{\sin w \sqrt{k} t}\right)^{2}+ \\
& \sin w \sqrt{k-1} t \sin w \sqrt{k}(t-\tau)\left[\left(x_{10} x_{2 \tau}-x_{20} x_{1 \tau}\right) .\right. \\
& \left.\sin w \sqrt{k} t-\left(x_{10} x_{20}-x_{20} x_{1}^{\prime \prime}\right) \sin w \sqrt{k} \tau\right]+ \\
& \left(x_{1 \tau} x_{2}^{\prime \prime}-x_{2 \tau} x_{1}^{\prime \prime}\right) \sin w \sqrt{k-1}(t-\tau) \sin w \sqrt{k} \tau \sin w \sqrt{k} t
\end{aligned}
$$

with

$$
\eta=\frac{-m w \sin (w \sqrt{k} t)}{\hbar \sqrt{k} \sin (w \sqrt{k} \tau) \sin (w \sqrt{k}(t-\tau))}
$$

For $\theta=0$, equation (8) becomes

$W_{\theta}^{H}\left(x_{0}, 0 ; x_{\tau}, \tau ; x, t\right)=\left[\frac{(2 \pi \hbar \sin (w \tau)) \sin (w(t-\tau))}{m w \sin (w t)}\right]^{\frac{-1}{2}} \exp \left\{\frac{-\mathrm{mwcos}(\mathrm{wt})}{2 \hbar \sin (\mathrm{w} \tau) \sin (\mathrm{w}(\mathrm{t}-\tau))}\left[\xi_{1}^{2}\left(\mathrm{x}_{10}, 0 ; \mathrm{x}_{1 \tau}, \tau ; \mathrm{x}_{1}^{\prime \prime}\right)+\xi_{2}^{2}\left(\mathrm{x}_{20}, 0 ; \mathrm{x}_{2 \tau}, \tau ; \mathrm{x}_{2}^{\prime \prime}\right)\right]\right\}$

Equation (13) is the probability density of the harmonic oscillator in two dimensional spaces [1]. With the help of the factorization theorem, the solution of (8) is expressed as follows:

$$
W_{\theta}^{H}=\frac{\exp \left\{-\frac{1}{\hbar} S_{\theta}^{H 1}\left(x_{\tau}, \tau ; x^{\prime \prime}, t\right)\right\}}{\exp \left\{-\frac{1}{\hbar} S_{\theta}^{H 1}\left(x_{0}, 0 ; x^{\prime \prime}, t\right)\right\}} Z_{\theta}\left(x_{0}, 0 ; x_{\tau}, \tau\right)
$$

where

$$
Z_{\theta}\left(x_{0}, 0, x_{\tau}, \tau\right)=\int_{0} \exp \left\{-\frac{1}{\hbar} \int_{0}^{t} V\left(x_{\tau}, \tau\right) d w\left(x_{\tau}\right)\right\}
$$

and

$$
\begin{gathered}
V\left(x_{\tau}, \tau\right)=\frac{2}{\hbar} \frac{\partial S_{\theta}^{H 1}\left(x_{\tau}, \tau ; x^{\prime \prime}, t\right)}{\partial \tau}-\frac{1}{2 m \hbar}\left[\frac{\partial S_{\theta}^{H 1}\left(x_{\tau}, \tau ; x^{\prime \prime}, t\right)}{\partial x_{1 \tau}}+\frac{\partial S_{\theta}^{H 2}\left(x_{\tau}, \tau ; x^{\prime \prime}, t\right)}{\partial x_{1 \tau}}+\frac{\partial S_{\theta}^{H 1}\left(x_{\tau}, \tau ; x^{\prime \prime}\right)}{\partial x_{2 \tau}}+\frac{\partial S_{\theta}^{H} 2\left(x_{\tau}, \tau, x^{\prime \prime}, t\right)}{\partial x_{2 \tau}}\right] \\
+\frac{m}{\hbar}\left[\frac{\partial S_{\theta}^{H}{ }^{2}\left(x_{\tau}, \tau ; x^{\prime \prime}, t\right)}{\partial x_{1 \tau}}\left(\dot{x}_{1 \tau}+\frac{1}{m} \frac{\partial S_{\theta}^{H 1}\left(x_{\tau}, \tau ; x^{\prime \prime}, t\right)}{\partial x_{1 \tau}}\right)+\frac{\partial S_{\theta}^{H 2}\left(x_{\tau}, \tau ; x^{\prime \prime}, t\right)}{\partial x_{2 \tau}}\left(\dot{x}_{2 \tau}+\frac{1}{m} \frac{\partial S_{\theta}^{H 1}\left(x_{\tau}, \tau ; x, t\right)}{\partial x_{2 \tau}}\right)\right]+ \\
\frac{\hbar^{2}}{2 m}\left[\left(\frac{\partial S_{\theta}^{H 1}\left(x_{\tau}, \tau ; x^{\prime \prime}, t\right)}{x_{1 \tau}}\right)^{2}+\left(\frac{\partial S_{\theta}^{H 1}\left(x_{\tau}, \tau ; x^{\prime \prime}, t\right)}{\left.x_{2 \tau}\right)}\right)^{2}\right] \\
S_{\theta}^{H 1}\left(x_{\tau}, \tau ; x^{\prime \prime}, t\right)=\frac{m w}{2 \sqrt{k} \sin (w \sqrt{k} T)}\left[\left(x_{1 \tau}^{2}+x_{2 \tau}^{2}+x_{1}^{\prime \prime 2}+x_{2}^{\prime \prime 2}\right) \cos (w \sqrt{k} T)+\right] \\
2\left(x_{1 \tau} x_{1}^{\prime \prime}+x_{2 \tau} x_{2^{\prime \prime}}\right) \cos (w \sqrt{k-1} T) \\
S_{\theta}^{H 2}\left(x_{\tau}, \tau ; x^{\prime \prime}, t\right)=\frac{m w}{\sqrt{k} \sin (w \sqrt{k} T)}\left[\left(x_{1} x_{2}^{\prime \prime}-x_{2 \tau} x_{1}^{\prime \prime}\right) \sin (w \sqrt{k-1} T)\right]
\end{gathered}
$$


From (14), and with the help of the transformation leading to transition from Euclidian quantum mechanics to statistical physics, the solution of Bloch equation yields:

$$
Y_{\theta}^{H}\left(x_{\tau}, x, \hbar \lambda\right)=\frac{m w}{2 \pi \hbar \sqrt{k} \sinh (w \hbar \sqrt{k} \lambda)} \exp \left\{-\frac{m w}{2 i \sqrt{k} \hbar \sinh w \sqrt{k}(t-\tau)}\left[\begin{array}{c}
\left(x_{1 \tau}^{2}+x_{2 \tau}^{2}+x_{1}^{\prime 2}+x_{2}^{\prime 2}\right) \cosh (w \hbar \sqrt{k} \lambda) \\
-2\left(x_{1 \tau} x_{1}^{\prime \prime}+x_{2 \tau} x_{2}^{\prime \prime}\right) \cosh (w \hbar \sqrt{k-1} \lambda)
\end{array}\right]\right\}
$$

Then the partition function of the harmonic oscillator in non-commutative space is derived[19, 20]

$$
Z_{\theta}^{H}=\frac{1}{2[\cosh (\hbar w \sqrt{k} \lambda)-\cosh (w \hbar \sqrt{k-1} \lambda)]}
$$

The entropy, the internal energy and the specific heat capacity are respectively expressed by the following relations:

$$
\begin{gathered}
S_{\theta}^{H}(T)=\frac{w \hbar \lambda}{k_{B} T \sigma}-\ln 2 \sigma \\
E_{\theta}^{H}(T)=-\frac{w \hbar \lambda}{k_{B} \sigma} \\
\mathrm{C}_{\theta \mathrm{V}}^{\mathrm{H}}=\frac{\mathrm{w} \hbar}{\mathrm{k}_{\mathrm{B}}(\mathrm{T} \sigma)^{2}}\left[\frac{2 \mathrm{w} \hbar \sqrt{\mathrm{k}(\mathrm{k}-1)}}{\mathrm{k}_{\mathrm{B}}} \mathrm{a}-\frac{\mathrm{w} \hbar}{\mathrm{k}_{\mathrm{B}}}(\sqrt{\mathrm{k}(\mathrm{k}-1)}+\mathrm{k}) \mathrm{b}\right]
\end{gathered}
$$

where:

$$
\begin{gathered}
\sigma=\cosh \left(\frac{w \hbar \sqrt{k}}{k_{B} T}\right)-\cosh \left(\frac{w \hbar \sqrt{k-1}}{k_{B} T}\right) \\
\lambda=\sqrt{k} \sinh \left(\frac{w \hbar \sqrt{k}}{k_{B} T}\right)-\sqrt{k-1} \sinh \left(\frac{w \hbar \sqrt{k-1}}{k_{B} T}\right) \\
a=\cosh \left(\frac{w \hbar \sqrt{k}}{k_{B} T}\right) \cosh \left(\frac{w \hbar \sqrt{k-1}}{k_{B} T}\right)-1 \\
b=\sinh \left(\frac{w \hbar \sqrt{k}}{k_{B} T}\right) \sinh \left(\frac{w \hbar \sqrt{k-1}}{k_{B} T}\right)
\end{gathered}
$$

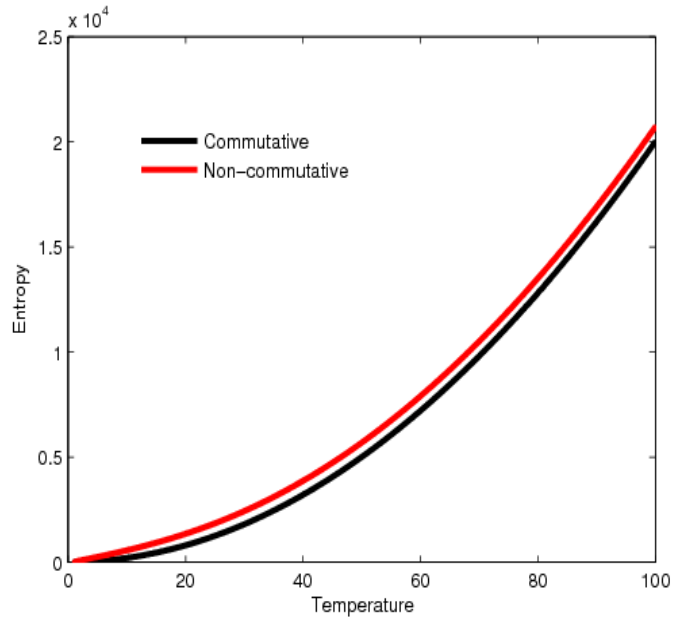

Figure 1. Entropy versus temperature in commutative and noncommutative space.

The curves plotted respectively in Figs. 1 to 3 show the influence of temperature on the behavior of the thermodynamic parameters when considering simultaneously the commutative and the non-commutative space. It is found that the non-commutative parameter is depicted for increasing value of the entropy [21,22] and the internal energy.

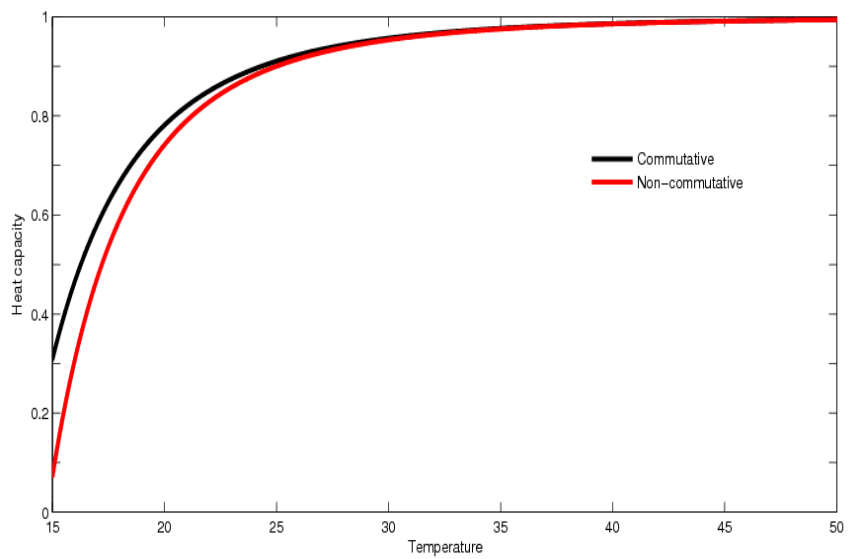

Figure 2. Heat capacity versus temperature in commutative and noncommutative space. 


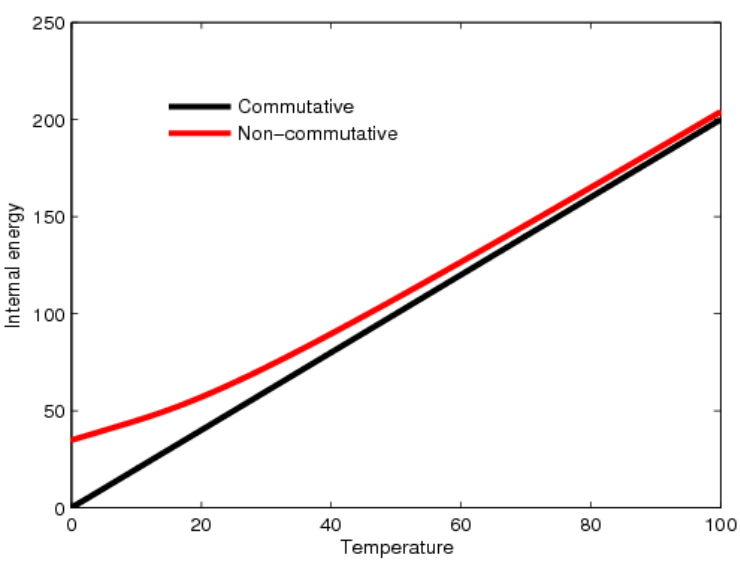

Figure 3. Internal energy versus temperature in commutative and noncommutative space.

\section{Conclusion}

In this work, we present the Jacobi's second equality in the form of stochastic equation of harmonic oscillator in non-commutative space. The results show that there is no modification on the structure of the Fokker-Planck equation; i.e. the factorization theorem is conserved in commutative and non-commutative space. Moreover, the noncommutative parameter is depicted for increasing value of the entropy and internal energy of the system.

\section{References}

[1] M.Tchoffo and A.A.Beilinson, "path integral approach to the kinematical Brownian motion due to random canonical transformation.” Far East J.Appl.Math 36, 1, 2009, pp.25-36

[2] S.Massou, M.Tchoffo, "Factorization of the solution of Fokker-planck equation," Far East J.Appl.Math volume 10, 2, 2012, pp.161-170.

[3] M.Tchoffo, J.C. Ngana et al "Kinematical Brownian motion and time dependent entropy", Far east J. vol 68, 1, 2012, pp. $21-28$

[4] Massou. S, Moussiliou. S and M.Tchoffo ,"stochastic chaos in a class of Fokker-equation derived from population dynamic", Applied Physics research 4, 3, 2012.

[5] LY.Chen, P.L.Nash, "Stochastic Simulations of Clusters: Quantum Methods in Flat and Curved Spaces", journal of chemical physics 121, 9, 2004.

[6] Yu L klimontovich, "Nonlinear Brownian motion physicsUspekhi” 37, 8, 1994, pp.737-767.
[7] J.L.F. Barbón, "Pertubing The groundring of 2D String theory”, Int.J. Mod.Phys. A 07, 7579, 1992.

[8] Achim Kempf, "Quantum integration on non-commutative geometries", polish academy of science 40, 1997.

[9] S. Paul Smith, "integral non-commutative space", journal of algebra 246, 2001, pp.793-810.

[10] Hugo Garcia, octavio Obregon et al, "Non-commutativity in gravity, topological gravity and cosmology" J of phys (conference serie) 24, 2005, pp.203-212.

[11] J.C Ferrando, L.M.Sanchez, R. R. Mountain: "A survey on recent advances on the nikodim boundeness theorem and space of simple function" J.math 34, 2004, pp.139-172.

[12] S. A. A Ghorashi, M.Bagheri Harouni, "Decoherence of quantum Brownian motion in non commutative space" physic letters A 377, 2013, pp.952-956.

[13] V.P.Belavkin, "quantum ito B-algebras, their classification and decomposition", polish academy of science 43, 1998 .

[14] wlod ziemerz bryz and victor bryc, "strong mixing coefficient for non-commutative Gaussian process", proc Amer.math. soc 132, 2004, pp.523-534.

[15] R.Vilela Mendes, "Quantum mechanics and noncommutative space", physics letters A 210, 1996, pp.232240 .

[16] A. Jahan, "non commutative harmonic oscillator at finite temperature", a path integral approach, Brazilian journal of physics, 37, 2007.

[17] Branko Dragovich, Zoran Rakic, "path integral in noncommutative quantum mechanics", Theoretical and math phys $140,3,2004$. pp.1299-1308.

[18] M.Chaichian, A.Tureanu, A.Zahahi "solution of the stochastic Langevin equations for clustering of particles in random flows in terms of the wiener path integral", Phys.Rev. E 81, 066309, 2010 .

[19] Iraj Jabbari, Akbar Jahan, and Zafar Riaziturk "Partition function of the harmonic oscillator on a non commutative plane", J.phys 33, 2009, pp.149-154.

[20] Jean Zinn-Justin, "intégrale de chemin en mécanique quantique ", (EDP Sciences/CNRS editions, 2003).

[21] A.E. Bernadini and O.Bertolami, "Probing phase-space non-commutativity through quantum beating, missing information and the thermodynamic limit", Phys.Rev A 88, 012101, 2013.

[22] Adil Belhaj, M.chabab et al, "on non-commutative Black holes and their thermodynamics in arbitrary dimension", The African review of physics 8, 0017, 2013. 